\title{
The Associations of Active Travel to School with Physical Activity and Screen Time Among Adolescents: Do Individual and Parental Characteristics Matter?
}

\author{
Caihong Huang ( $\sim$ rainbow357@126.com ) \\ Hubei University of Technology \\ Aamir Raoof Memon \\ Victoria University \\ Chaomei Liang \\ Library of Beijing Sport University \\ Jin-Tao Hong \\ Shanghai Research Institute of Sports Science \\ Youliang Lin \\ Wuhan University of Technology \\ Si-Tong Chen \\ Victoria University
}

\section{Research article}

Keywords: active travel, age, geographical location, parents, physical activit, school-aged children, screen time, sex

Posted Date: August 18th, 2020

DOI: https://doi.org/10.21203/rs.3.rs-52361/v1

License: (c) (i) This work is licensed under a Creative Commons Attribution 4.0 International License. Read Full License

Version of Record: A version of this preprint was published at Frontiers in Public Health on December 15th, 2021. See the published version at https://doi.org/10.3389/fpubh.2021.719742. 


\section{Abstract}

Background: Little is known about the relationship of active travel to school (ATS) with physical activity (PA) and screen time (ST) by individual and parental characteristics among adolescents, especially in China. To address the research gap, this study aimed to explore the difference of sex, age, geographical location, parental occupation and education level in the relationship of ATS with PA and ST among students of grades 7-12 using cross-sectional data.

Methods: In 13 cities of Hubei province, China, students from 39 public schools were recruited to engage in the survey. In total, 5898 students (response rate $=89.6 \%$ ) were invited into this study. Participants were required to report their ATS (including its types), PA and ST as well as sociodemographic information using validated questionnaire. Descriptive analyses were used to report the information of all variables. Regression models were used to analyze the relationships of ATS and its types with PA and ST.

Results: In a total of 4128 participants (boy: $50.9 \%$; younger adolescents: $61.9 \%$ ) included in the final analysis, the proportion of those with ATS was $47.3 \%$. Regarding the types of ATS, walking accounted for over $30 \%$, while cycling was $13.2 \%$. Participants with ATS were more likely to have sufficient $\mathrm{PA}(\mathrm{OR}=1.26,95 \% \mathrm{Cl}$ : 1.14-1.39), especially among boys, younger adolescents and those with lower parental education level. However, ATS was not associated with ST ( $\mathrm{OR}=0.94,95 \% \mathrm{Cl}: 0.86-1.01)$. Participants with cycling had higher odds ratio of being physically active (OR for cycling $=1.47,95 \% \mathrm{Cl}$ : 1.27-1.70; OR for walking $=1.18,95 \% \mathrm{Cl}$ : 1.06-1.32). The association of ATS types with PA and ST differed by gender, age, geographical location and parental educational level as well as occupations.

Conclusions: ATS may be a useful approach to increase PA among adolescents, but this should be explained by individual and parental characteristics.

\section{Background}

A markedly ubiquitous international trend is that only a small proportion of adolescents fulfil the pervasively recognised physical activity (PA) and screen time (ST) guidelines [1-3]. Recent data based on objective measures indicate that just $44.1 \%$ of children and adolescents met the PA guidelines ( $\geq 60$ min of moderate to vigorous PA (MVPA)), while $39.3 \%$ of participants met the ST guidelines ( $<2$ hours per day) [4]. Conversely, meeting the PA and ST recommendations is correlated with more desired health outcomes [5-8]. For example, higher PA levels were conversely related to (and greater time spent in ST was positively related to) the odds ratio of being obese [9]. For more desirable health outcomes, concurrently promoting PA and discouraging ST among adolescents is an essential public health action.

Given the insufficient PA and higher ST among adolescents globally, researchers have sought effective interventions to change these harmful health behaviours [10, 11]. In order to more effectively promote PA and limit ST among adolescents, active travel to school (ATS) plays a significant role. ATS is an essential source of PA behavioural components among adolescents. Well-documented evidence has presented how ATS is positively correlated with PA among adolescents [12]. For example, Roth et al. [13] demonstrated that children and adolescents participating in walking or cycling (types of ATS) were more likely to meet the PA recommendation $(\mathrm{OR}=1.31)$. A further recent investigation of Ecuadorian students similarly demonstrated that participants engaged in ATS were approximately 3 times more likely to be engaged in sufficient PA as opposed to their peers [14]. Comparable results were established in another study conducted in the United Kingdom [15]. Overall, the extant research supports the position that ATS can promote PA among adolescents. However, concerning the relationship of ATS with ST among adolescents, an ambiguous relationship remains [12]. For example, certain researchers have posited that active travellers among adolescents had a reduced chance of spending time in ST [16-18], although this conclusion contrasted with other studies $[19,20]$. Collectively, the relationship of ATS with ST should be clarified further, drawing on the accumulated evidence. Through drawing on the available literature, ATS may pose an effective means of increasing PA and ST, albeit with the relationship between ATS and ST being ambiguous [12].

With the increasing importance of ATS on overall PA, Chinese researchers have been devoted to ATS-related studies among Chinese young people. For example, Sun et al. [21] explored the health benefits of ATS, including lower body mass index and lower odds of depressive symptoms among nationally representative Chinese samples. In a cross-sectional study, Yang et al. [22] reported that there was a decline in ATS among Chinese adolescents. Also, some recent studies identified the social or environmental factors of ATS [23,24]. Within the limited literature, few studies investigated the relationship of ATS with PA and ST. Gao et al. [25] reported that, using a pedometer device, male schoolchildren with more steps during before- and after- school time showed higher levels of activity time, while female schoolchildren who had more steps during after-school time reported high activity. In Hong Kong, Huang et al. [26] found that ATS was related to maintaining a relatively higher level of PA. However, little is known about the relationship of ATS with ST among Chinese young people. Despite studies having presented evidence regarding the relationship of ATS with PA and ST, several research gaps still exist. One gap concerns the fact that the recent studies concerning ATS and PA, as well as ST, were based on adolescents from western countries [12, 21], whereas there is 
limited understanding about them with respect to Chinese adolescents. Since evidence suggests that Chinese adolescents are insufficiently active [22], it is anticipated that ATS offers the potential to enhance their PA based on the findings of western studies. Such a relationship has not been investigated conclusively among Chinese samples. Understanding the relationship of ATS with PA is conducive to designing efficient interventions for enhanced PA among Chinese adolescents. Additionally, the correlation between ATS with ST continues to be unclear; thus, this correlation is worth investigating through additional studies [12]. In this regard, replicating comparable studies is necessary to advance our knowledge of PA behaviour, which may also provide strategies for reducing ST among Chinese adolescents.

The ATS, PA, and ST are associated with the substantial differences in individual and parental characteristics among adolescents. For example, Yang et al. [22] reported that adolescent ATS was significantly higher among younger groups, participants with lower educational attainment, or those residing in rural areas. Certain studies in western countries established that socioeconomic status parameters, for example living geographical location, parental education, and working status, were linked with ATS among adolescents [13,15,17]. Such variables are associated with PA and ST among adolescents in accordance with the Social Ecological Model [27]. Given the correlations between ATS, PA, ST, and socio-demographic variables, the correlation of ATS with PA and ST among adolescents may vary according to their individual and parental characteristics. As suggested in the review by Lubans et al. [28], investigating the overall relationship of ATS with outcomes (e.g., fitness or lifestyles behaviour) ignores the wider personal or social level correlates of behaviour. Therefore, it is necessary to investigate the correlation of ATS with PA and ST further, in accordance with the individual and parental characteristics. Nevertheless, across the limited literature, less research has scrutinised the relationship of ATS with PA and ST in relation to sociodemographic factors. For instance, one study indicated that the relationship of ATS with PA was only significant among younger adolescents in Ecuador [14]. Another study found that sex is a potential moderator of the relationship between ATS and PA among children and adolescents [19]. Unfortunately, there is a dearth of relevant evidence in this regard from China, which restricts our comprehension of the relationship of ATS with PA and ST among Chinese adolescents. Furthermore, there is limited understanding concerning investigations into the role of parental characteristics in this relationship.

Certain parental characteristics, for example, education and occupation, are associated with school travel choices. For instance, higher parental educational attainment represents advantages in terms of income, with those parents potentially more likely to deliver their children at school using private vehicles $[13,16,19]$. Subsequently, this could affect their children's active lifestyles. Nonetheless, whether the relationship of ATS with PA and ST varies according to parental characteristics has not yet been investigated. This is necessary to advance the knowledge in this field and capture information regarding the finer practical implications for adolescents affected by various parental characteristics.

Therefore, as a means of contributing to resolving the evidence shortcomings in the extant literature and to develop an evidence foundation for PA- and ST-focused interventions for Chinese adolescents, this research aimed to analyse the relationship of ATS with PA and ST in accordance with the individual and parental characteristics among adolescents in China.

\section{Methods}

\section{Study design and research ethics}

The data collection method adopted in this cross-sectional study was a questionnaire survey, undertaken between May and June 2019, in Hubei province of China. Contact was made with the commission of education in 13 cities of Hubei province. Applying a convenience sampling method, we invited three public schools (one primary school, one middle school, and one high school) per city to participate in this survey. Through the administrative support provided by the commissions of education, 3rd to 12 th grade students in 39 public primary, middle, and high schools were selected across all the cities. Among the entire student sample obtained, 6583 students (aged 10 to 18 years) were invited to participate in the survey. Of these, 5898 responses with a complete self-reported questionnaire, providing a response rate of $89.6 \%$. The research protocol and procedure were approved by the Institutional Review Board (IRB) of the Wuhan University of Technology in March 2019. The student participants and their legal guardians provided written consent. The anonymity and confidentiality of participants was ensured in accordance with the Declaration of Helsinki.

\section{Measurements}

\section{Sociodemographic information}

The participants were asked, during their break time on schooldays, to provide self-reported data regarding sex ( 1 = boy, $2=$ girl), grade (from 4 to 12 ) and current living geographical locations ( 1 = urban, 2 = rural). A self-reported questionnaire was implemented to collect 
information from parents, including the parental educational attainment (less than college/university; college/university or higher), and occupation (office worker or manual laborer). The parents completed the paper-based questionnaire at home using a pencil.

\section{Exposures (ATS)}

A single-item question was included for measuring the ATS, which asked the participants: 'In the last 7 days, how did you usually get to your school?' [21]. This measure has been used in previously published studies [21]. Participants were asked to indicate which of the travel modes represented their principal means of travelling to school and returning home. They were only given the option to select one principal transport mode, and data relating to multimode journeys (walking to or from public transport hubs, for example) was not obtained. Participants could select the following options as their responses: 1) walking independently; 2) cycling independently; 3) walking with parents or guardians; 4) cycling with parents or guardians; 5) taking school bus; 6) delivered by parents using automobile/private car/other motorized instruments; 7) other public transportation, such as public bus/underground/taxi. Overall, participants selecting options from 1 to 4 were considered active, whereas others were considered passive. According to the type of ATS, participants selecting 1 and 3 were regarded as walking; 2 and 4 were regarded as cycling; 5 were regarded as using school bus; 6 were regarded as delivered by parents; and 7 were regarded as using public transportation.

\section{Outcomes (PA and ST)}

PA was measured using the items derived from the Health Behavior School-aged Children (HBSC) questionnaire, which has acceptable validity and reliability in the Chinese context [29]. Two items collected the information on PA, namely, 1) 'How many days did you engage in moderate to vigorous PA (MVPA) for at least 60 minutes on weekdays over the past week?', with responses: $0=$ none, $1=1$ day, $2=2$ days, 3 $=3$ days, $4=4$ days, $5=5$ days; and 2 ) 'How many days did you engage in MVPA for at least 60 minutes during the weekend over the past week?', where responses included: $0=$ none, $1=1$ day, $2=2$ days. Consistent with the Canadian 24-Hour Movement Guideline [4], sufficient PA in accordance with the definition of fulfilling the MVPA recommendation was equivalent to the participants reporting 60 minutes of MVPA daily for seven days.

The following items of the HBSC questionnaire were used to obtain information relating to ST [29]: 1) 'How many hours did you spend watching TV or movies during your leisure time on weekdays and on the weekend over the past week?' (responses: $1=$ none, $2=$ around half an hour, 3 = around one hour, 4 = around two hours, 5 = around three hours of more); 2 ) 'How many hours did you spend playing computer games during your leisure time on weekdays and on the weekend over the past week?' (responses: same as the above item); and 3) 'How many hours did you spend on activities using electronic screen-based devices during your leisure time on weekdays and on the weekend over the past week?' (responses: same as the above item). Consistent with the Canadian 24-Hour Movement Guidelines [8], limited ST was considered to be daily screen time of participants-including time watching TV/movies, playing computer games, and using electronic screen-based devices-of less than 2 hours per day.

\section{Statistical analysis}

Prior to initiating the formal analysis process, all responses with missing data were omitted from the sample. We opted to concentrate our analysis on adolescents (grades 7-12) only, with data pertaining to further school grades being excluded. Ultimately, the final sample size, included in the analysis, was 4128. Descriptive statistical analysis was applied to report the percentage of sociodemographic variables (e.g. sex, age, geographic location, parental occupation and education), exposures (ATS: passive versus active; its types: school bus, delivery by parents, or public transportation as passive, or walking or cycling as active) and outcomes (PA: sufficient versus insufficient; ST: limited versus excessive). Chi square test was performed to investigate the difference in PA and ST by ATS (its types), alongside the individual as well as parental characteristics. Logistic regression was undertaken to analyse the relationship of ATS and its types (exposure) with PA and ST (outcome). All variables were incorporated into the regression analysis as categorical variables. This research presents the logistic regression results as odds ratios (ORs) with $95 \%$ confidence intervals (Cls). When examining the relationship of ATS and its types with PA and ST, all sociodemographic variables were adjusted in the models, and $p<0.05$ was established as the level of statistical significance. The statistical analyses procedures were performed using SPSS 24.0 (IBM Corp, Chicago, IL, USA).

\section{Results}

Table 1 presents the characteristics of participants in this study. Of all the included participants $(n=4128)$, boys accounted for $50.9 \%$. Younger adolescents accounted for over $60 \%$ of the participants. Over $70 \%$ of the participants lived in urban areas. Approximately $70 \%$ of participants had parents who were office worker or had an education degree of less than college/university. The proportion of participants using passive mode of ATS was $52.7 \%$. Regarding the types of ATS, participants who walked to school accounted for $34.1 \%$ (the largest proportion), while participants using the school bus made up the smallest proportion (0.8\%). The prevalence of sufficient PA and limited ST was $17.3 \%$ and $67.9 \%$, respectively. 
Characteristics of participants in this study

\begin{tabular}{|c|c|c|c|}
\hline & & $\mathbf{n}$ & $\%$ \\
\hline Overall & & 4128 & 100 \\
\hline \multicolumn{4}{|l|}{ Sex } \\
\hline & Boy & 2101 & 50.9 \\
\hline & Girl & 2027 & 49.1 \\
\hline \multicolumn{4}{|c|}{ Age group } \\
\hline & Younger adolescent & 2555 & 61.9 \\
\hline & Older adolescent & 1573 & 38.1 \\
\hline \multicolumn{4}{|c|}{ Geographical location } \\
\hline & Urban & 3059 & 74.1 \\
\hline & Rural & 1069 & 25.9 \\
\hline \multicolumn{4}{|c|}{ Parental occupation } \\
\hline & Officer worker & 2881 & 69.8 \\
\hline & Manual laborer & 1247 & 30.2 \\
\hline \multicolumn{4}{|c|}{ Parental education level } \\
\hline & Less than college/university & 2935 & 71.1 \\
\hline & College/university or higher & 1193 & 28.9 \\
\hline \multicolumn{4}{|c|}{ Travel to school } \\
\hline & Passive & 2175 & 52.7 \\
\hline & Active & 1953 & 47.3 \\
\hline \multicolumn{4}{|c|}{ Modes of travel to school } \\
\hline & School bus & 33 & 0.8 \\
\hline & Delivered by parents & 1102 & 26.7 \\
\hline & Public transportation & 1040 & 25.2 \\
\hline & Walking & 1408 & 34.1 \\
\hline & Cycling & 545 & 13.2 \\
\hline \multicolumn{4}{|l|}{ MVPA* $^{*}$} \\
\hline & Insufficient & 3414 & 82.7 \\
\hline & Sufficient & 714 & 17.3 \\
\hline \multicolumn{4}{|l|}{$S T \star *$} \\
\hline & Excessive & 1325 & 32.1 \\
\hline & Limited & 2803 & 67.9 \\
\hline
\end{tabular}

In Table 2, the results of the difference in PA and ST by ATS and its types are presented. Participants with active ATS showed a higher percentage of sufficient PA compared to their counterparts $(20.1 \%$ vs. $14.8 \%, p=0.000)$. Participants taking cycling as ATS mode had the highest percentage of sufficient PA compared to others $(23.0 \%, p=0.000)$. There was no significant difference in the levels of limited ST among participants with different ATS groups. Participants taking the school bus as their mode of ATS had the lowest percentage of limited ST compared to those using other types of ATS $(53.3 \%, p=0.000)$. 
Table 2

Difference of PA and ST by ATS and its types

\begin{tabular}{|c|c|c|c|c|}
\hline & Sufficient PA ( $n=714)$ & \multirow[t]{2}{*}{$p$} & Limited ST $(n=2803)$ & \multirow[t]{2}{*}{$p$} \\
\hline & $\%$ & & $\%$ & \\
\hline \multicolumn{5}{|l|}{ ATS } \\
\hline Passive & 14.8 & \multirow[t]{2}{*}{0.000} & 68.4 & \multirow[t]{2}{*}{0.213} \\
\hline Active & 20.1 & & 67.3 & \\
\hline \multicolumn{5}{|l|}{ Types of ATS } \\
\hline School bus & 15.2 & \multirow[t]{5}{*}{0.000} & 53.3 & \multirow[t]{5}{*}{0.000} \\
\hline Delivered by parents & 15.9 & & 73.0 & \\
\hline Public transportation & 13.6 & & 64.0 & \\
\hline Walking & 19.0 & & 69.5 & \\
\hline Cycling & 23.0 & & 61.7 & \\
\hline
\end{tabular}

The results of the difference of PA and ST by ATS and its types as well as individual characteristics are shown in Table 3 . Regardless of sex, and living geographical location, a difference of PA was observed between the passive and active ATS groups as well as the types of ATS (all p < 005). However, there was no significant difference of PA between the passive and active ATS groups among older adolescents $(p=0.116)$. Further, a significant difference was observed across groups of different types of ATS irrespective of sex, age, and living geographical location for limited ST. However, there was no significant different of ST across groups of ATS except for younger boys ( $p=$ 0.000). 
Table 3

Difference of PA and ST by ATS and its types as well as individual characteristics

\begin{tabular}{|c|c|c|c|c|c|c|c|c|}
\hline & \multicolumn{4}{|c|}{ Sufficient PA $(n=714)$} & \multicolumn{4}{|c|}{ Limited ST $(n=2803)$} \\
\hline & $\%$ & $p$ & $\%$ & $p$ & $\%$ & $p$ & $\%$ & $p$ \\
\hline & Boy & & Girl & & Boy & & Girl & \\
\hline \multicolumn{9}{|l|}{ ATS } \\
\hline Passive & 17.8 & \multirow[t]{2}{*}{0.000} & 12.3 & \multirow[t]{2}{*}{0.040} & 66.5 & \multirow[t]{2}{*}{0.562} & 70.0 & \multirow[t]{2}{*}{0.621} \\
\hline Active & 24.6 & & 14.1 & & 65.7 & & 69.4 & \\
\hline \multicolumn{9}{|l|}{ Types of ATS } \\
\hline School bus & 18.2 & \multirow[t]{5}{*}{0.000} & 10.8 & \multirow[t]{5}{*}{0.010} & 58.2 & \multirow[t]{5}{*}{0.000} & 45.9 & \multirow[t]{5}{*}{0.000} \\
\hline Delivered by parents & 19.6 & & 13.3 & & 70.8 & & 74.5 & \\
\hline Public transportation & 16.3 & & 10.9 & & 63.1 & & 64.9 & \\
\hline Walking & 24.5 & & 13.4 & & 68.7 & & 70.3 & \\
\hline \multirow[t]{2}{*}{ Cycling } & 24.9 & & 17.5 & & 60.5 & & 65.2 & \\
\hline & \multicolumn{2}{|c|}{ Younger adolescents } & \multicolumn{2}{|c|}{ Older adolescents } & \multicolumn{2}{|c|}{ Younger adolescents } & \multicolumn{2}{|c|}{ Older adolescents } \\
\hline \multicolumn{9}{|l|}{ ATS } \\
\hline Passive & 17.4 & \multirow[t]{2}{*}{0.000} & 11.6 & \multirow[t]{2}{*}{0.116} & 72.0 & \multirow[t]{2}{*}{0.010} & 63.9 & \multirow[t]{2}{*}{0.559} \\
\hline Active & 23.2 & & 13.2 & & 69.2 & & 63.1 & \\
\hline \multicolumn{9}{|l|}{ Types of ATS } \\
\hline School bus & 12.5 & \multirow[t]{5}{*}{0.000} & 17.3 & \multirow[t]{5}{*}{0.002} & 55.0 & \multirow[t]{5}{*}{0.000} & 51.9 & \multirow[t]{5}{*}{0.000} \\
\hline Delivered by parents & 17.3 & & 12.6 & & 73.5 & & 71.8 & \\
\hline Public transportation & 17.7 & & 10.9 & & 69.8 & & 60.0 & \\
\hline Walking & 21.9 & & 11.0 & & 70.9 & & 65.5 & \\
\hline \multirow[t]{2}{*}{ Cycling } & 27.8 & & 16.6 & & 63.6 & & 59.2 & \\
\hline & \multicolumn{2}{|l|}{ Urban } & Rural & & Urban & & Rural & \\
\hline ATS & & & & & & & & \\
\hline Passive & 14.9 & 0.000 & 14.6 & 0.000 & 68.5 & 0.050 & 68.0 & 0.414 \\
\hline Active & 19.5 & & 21.8 & & 66.5 & & 69.4 & \\
\hline Types of ATS & & & & & & & & \\
\hline School bus & 16.9 & 0.000 & 12.1 & 0.000 & 55.9 & 0.000 & 48.5 & 0.000 \\
\hline Delivered by parents & 16.2 & & 14.9 & & 73.3 & & 72.2 & \\
\hline Public transportation & 13.4 & & 14.3 & & 63.9 & & 64.2 & \\
\hline Walking & 17.9 & & 21.9 & & 68.7 & & 71.8 & \\
\hline Cycling & 23.5 & & 21.7 & & 61.0 & & 63.6 & \\
\hline
\end{tabular}

In Table 4, more participants with an active ATS had sufficient PA regardless of parental education levels compared to those with a passive ATS (less than college/university: $20.7 \%>14.6 \%, p<0.001$; college/university or higher: $18.7 \%>15.2 \%, p=0.006$ ), and particularly, participants who selected cycling had the highest percentages compared with the other ATS types. In terms of limited ST, participants who were delivered by parents had the highest percentages (less than college/university: $70.6 \%, p<0.001$; college/university or higher: $77.9 \%, p$ $=0.004$ ). Similar to the parental education level groups, more participants using active form of travel to school had sufficient PA regardless 
of their parent's occupations compared with those with passive ATS (office worker: $20.4 \%>14.9 \%$, manual laborer: $19.6 \%>14.6 \%$, both $p<$ 0.001), and participants using cycling had the highest percentages compared with other ATS types (both $23.0 \%$, both $p<0.001$ ).

Participants who were delivered by parents had the highest percentages of limited ST compared to those with other ATS types (office worker: $74.6 \%$, manual laborer: $68.6 \%$, both $p<0.001$ ).

Table 4

Difference of PA and ST by travel to school and its types as well as parental characteristics

\begin{tabular}{|c|c|c|c|c|c|c|c|c|}
\hline & Sufficient PA & & & & Limited ST & & & \\
\hline & $\%$ & $p$ & $\%$ & $p$ & $\%$ & $p$ & $\%$ & $p$ \\
\hline $\begin{array}{l}\text { Parental } \\
\text { education }\end{array}$ & $\begin{array}{l}\text { Less than } \\
\text { college/university }\end{array}$ & & $\begin{array}{l}\text { College/university } \\
\text { or higher }\end{array}$ & & $\begin{array}{l}\text { Less than } \\
\text { college/university }\end{array}$ & & $\begin{array}{l}\text { College/university } \\
\text { or higher }\end{array}$ & \\
\hline \multicolumn{9}{|l|}{ ATS } \\
\hline Passive & 14.6 & \multirow[t]{2}{*}{0.000} & 15.2 & \multirow[t]{2}{*}{0.006} & 65.5 & \multirow[t]{2}{*}{0.819} & 75.4 & \multirow[t]{2}{*}{0.076} \\
\hline Active & 20.7 & & 18.7 & & 65.2 & & 72.7 & \\
\hline \multicolumn{9}{|l|}{ Types of ATS } \\
\hline School bus & 13.6 & \multirow[t]{5}{*}{0.000} & 18.2 & \multirow[t]{5}{*}{0.005} & 45.8 & \multirow[t]{5}{*}{0.000} & 66.7 & \multirow[t]{5}{*}{0.004} \\
\hline $\begin{array}{l}\text { Delivered by } \\
\text { parents }\end{array}$ & 15.8 & & 16.0 & & 70.6 & & 77.9 & \\
\hline $\begin{array}{l}\text { Public } \\
\text { transportation }\end{array}$ & 13.5 & & 14.0 & & 61.1 & & 72.3 & \\
\hline Walking & 19.7 & & 17.3 & & 67.7 & & 74.1 & \\
\hline Cycling & 23.3 & & 22.3 & & 58.7 & & 69.1 & \\
\hline $\begin{array}{l}\text { Parental } \\
\text { occupation }\end{array}$ & Officer worker & & Manual laborer & & Officer worker & & Manual laborer & \\
\hline \multicolumn{9}{|l|}{ ATS } \\
\hline Passive & 14.9 & \multirow[t]{2}{*}{0.000} & 14.6 & \multirow[t]{2}{*}{0.000} & 70.3 & \multirow[t]{2}{*}{0.833} & 63.7 & \multirow[t]{2}{*}{0.205} \\
\hline Active & 20.4 & & 19.6 & & 70.1 & & 61.6 & \\
\hline \multicolumn{9}{|l|}{ Types of ATS } \\
\hline School bus & 16.9 & \multirow[t]{5}{*}{0.000} & 11.1 & \multirow[t]{5}{*}{0.000} & 60.0 & \multirow[t]{5}{*}{0.000} & 37.0 & \multirow[t]{5}{*}{0.000} \\
\hline $\begin{array}{l}\text { Delivered by } \\
\text { parents }\end{array}$ & 15.2 & & 17.7 & & 74.6 & & 68.6 & \\
\hline $\begin{array}{l}\text { Public } \\
\text { transportation }\end{array}$ & 14.4 & & 11.8 & & 65.8 & & 59.9 & \\
\hline Walking & 19.3 & & 18.4 & & 71.8 & & 64.8 & \\
\hline Cycling & 23.0 & & 23.0 & & 65.7 & & 52.8 & \\
\hline
\end{tabular}

In Table 5, the results from the logistic regression model revealing the relationships of ATS with PA and ST are shown. Overall, participants with ATS were more likely to have sufficient PA (OR $=1.26,95 \% \mathrm{Cl}$ : 1.14-1.39). In examining the relationships of ATS with PA and ST by sex, only boys with ATS had a higher chance of being sufficiently active $(\mathrm{OR}=1.41,95 \% \mathrm{Cl}: 1.24-1.61)$, compared with their counterparts. Significant relationships were also observed in participates of the younger age group $(\mathrm{OR}=1.35,95 \% \mathrm{Cl}: 1.20-1.52)$ and those whose parental education level were less than college/university $(O R=1.30,95 \% \mathrm{Cl}: 1.16-1.47)$. Regardless of geographical location and parental occupation, participants with ATS were more likely to have sufficient PA (all OR >1.20). However, participants with ATS showed a nonsignificant relationship with ST. 
Table 5

The relationships of travel (modes) to school with MVPA and ST by different characteristics

\begin{tabular}{|c|c|c|c|c|c|c|c|c|c|c|c|c|}
\hline & \multicolumn{6}{|c|}{ MVPA } & \multicolumn{6}{|l|}{ ST } \\
\hline & OR & \multicolumn{2}{|c|}{$95 \% \mathrm{Cl}$} & $\mathrm{OR}$ & \multicolumn{2}{|c|}{$95 \% \mathrm{Cl}$} & OR & \multicolumn{2}{|c|}{$95 \% \mathrm{Cl}$} & OR & \multicolumn{2}{|c|}{$95 \% \mathrm{Cl}$} \\
\hline Active & 1.26 & 1.14 & 1.39 & & & & 0.94 & 0.86 & 1.01 & & & \\
\hline \multirow[t]{2}{*}{ Passive } & Ref & & & & & & Ref & & & & & \\
\hline & Boys & & & Girls & & & Boys & & & Girls & & \\
\hline Active & 1.41 & 1.24 & 1.61 & 1.05 & 0.90 & 1.23 & 0.93 & 0.83 & 1.03 & 0.94 & 0.84 & 1.06 \\
\hline \multirow[t]{2}{*}{ Passive } & Ref & & & Ref & & & Ref & & & Ref & & \\
\hline & \multicolumn{3}{|c|}{ Younger adolescents } & \multicolumn{3}{|c|}{ Older adolescents } & \multicolumn{3}{|c|}{ Younger adolescents } & \multicolumn{3}{|c|}{ Older adolescents } \\
\hline Active & 1.35 & 1.20 & 1.52 & 1.07 & 0.88 & 1.29 & 0.91 & 0.82 & 1.01 & 0.98 & 0.86 & 1.12 \\
\hline \multirow[t]{2}{*}{ Passive } & Ref & & & Ref & & & Ref & & & Ref & & \\
\hline & Urban & & & Rural & & & Urban & & & Rural & & \\
\hline Active & 1.23 & 1.09 & 1.38 & 1.40 & 1.15 & 1.70 & 0.89 & 0.81 & 0.98 & 0.91 & 1.07 & 1.25 \\
\hline \multirow[t]{2}{*}{ Passive } & Ref & & & Ref & & & Ref & & & Ref & & \\
\hline & \multicolumn{3}{|c|}{ Less than college/university } & \multicolumn{3}{|c|}{ College/university or higher } & \multicolumn{3}{|c|}{ Less than college/university } & \multicolumn{3}{|c|}{ College/university or higher } \\
\hline Active & 1.30 & 1.16 & 1.47 & 1.16 & 0.96 & 1.39 & 0.98 & 0.89 & 1.07 & 0.84 & 0.72 & 0.99 \\
\hline \multirow[t]{2}{*}{ Passive } & Ref & & & Ref & & & Ref & & & Ref & & \\
\hline & \multicolumn{3}{|c|}{ Officer worker } & \multicolumn{3}{|c|}{ Manual laborer } & \multicolumn{3}{|c|}{ Officer worker } & \multicolumn{3}{|c|}{ Manual laborer } \\
\hline Active & 1.26 & 1.11 & 1.41 & 1.26 & 1.05 & 1.51 & 0.95 & 0.86 & 1.05 & 0.90 & 0.78 & 1.04 \\
\hline Passive & Ref & & & Ref & & & Ref & & & Ref & & \\
\hline
\end{tabular}

Table 6 shows the results from regression model for the relationships of types of ATS with PA and ST by individual and parental characteristics. In general, participants who selected walking or cycling were more likely to have sufficient PA overall, in boy, younger adolescents, and urban participants, and those who had parents with less than college/university education level or office worker occupation. Conversely, cycling had a likelihood of increasing the sufficient PA among girls, older adolescents, or those who had parents with a higher education level (college/university or higher) or manual laborer. With reference to the relationship of types of ATS with ST, participants with walking $(\mathrm{OR}=1.17,95 \% \mathrm{Cl}$ : $1.05-1.30)$ and delivered by parents $(\mathrm{OR}=1.34,95 \% \mathrm{Cl}: 1.19-1.50)$ were more likely to have limited ST. Significant relationships were also found among boys and girls, older adolescents, those living rural areas, those having parents with less than college/university education level, or with office workers. 
Table 6

The relationships of types of active travel to school with MVPA and ST by different characteristics

\begin{tabular}{|c|c|c|c|c|c|c|c|c|c|c|c|c|}
\hline & \multicolumn{6}{|c|}{ MVPA } & \multicolumn{6}{|l|}{ ST } \\
\hline & OR & \multicolumn{2}{|l|}{$95 \% \mathrm{Cl}$} & OR & \multicolumn{2}{|c|}{$95 \% \mathrm{Cl}$} & OR & \multicolumn{2}{|l|}{$95 \% \mathrm{Cl}$} & OR & \multicolumn{2}{|c|}{$95 \% \mathrm{Cl}$} \\
\hline Walking & 1.23 & 1.07 & 1.41 & & & & 1.17 & 1.05 & 1.30 & & & \\
\hline Cycling & 1.52 & 1.29 & 1.79 & & & & 0.88 & 0.77 & 0.99 & & & \\
\hline School bus & 1.06 & 0.59 & 1.89 & & & & 0.60 & 0.39 & 0.92 & & & \\
\hline $\begin{array}{l}\text { Delivered by } \\
\text { parents }\end{array}$ & 1.06 & 0.92 & 1.23 & & & & 1.34 & 1.19 & 1.50 & & & \\
\hline \multirow{2}{*}{$\begin{array}{l}\text { Public } \\
\text { transportation }\end{array}$} & \multicolumn{6}{|l|}{ Ref } & \multicolumn{6}{|l|}{ Ref } \\
\hline & \multicolumn{3}{|l|}{ Boys } & \multicolumn{3}{|l|}{ Girls } & \multicolumn{3}{|l|}{ Boys } & \multicolumn{3}{|l|}{ Girls } \\
\hline Walking & 1.41 & 1.18 & 1.69 & 0.99 & 0.79 & 1.23 & 1.16 & 1.01 & 1.34 & 1.17 & 1.01 & 1.37 \\
\hline Cycling & 1.57 & 1.29 & 1.91 & 1.47 & 1.07 & 2.00 & 0.83 & 0.70 & 0.98 & 0.97 & 0.77 & 1.23 \\
\hline School bus & 1.10 & 0.54 & 2.22 & 0.98 & 0.34 & 2.82 & 0.75 & 0.43 & 1.30 & 0.43 & 0.22 & 0.83 \\
\hline $\begin{array}{l}\text { Delivered by } \\
\text { parents }\end{array}$ & 1.07 & 0.88 & 1.32 & 1.01 & 0.81 & 1.27 & 1.25 & 1.06 & 1.48 & 1.42 & 1.21 & 1.66 \\
\hline \multirow{2}{*}{$\begin{array}{l}\text { Public } \\
\text { transportation }\end{array}$} & \multicolumn{3}{|l|}{ Ref } & \multicolumn{3}{|l|}{ Ref } & \multicolumn{3}{|l|}{ Ref } & \multicolumn{3}{|l|}{ Ref } \\
\hline & \multicolumn{3}{|c|}{ Younger adolescents } & \multicolumn{3}{|c|}{ Older adolescents } & \multicolumn{3}{|c|}{ Younger adolescents } & \multicolumn{3}{|c|}{ Older adolescents } \\
\hline Walking & 1.29 & 1.08 & 1.53 & 1.04 & 0.81 & 1.33 & 1.06 & 0.91 & 1.23 & 1.23 & 1.04 & 1.44 \\
\hline Cycling & 1.56 & 1.26 & 1.93 & 1.34 & 1.03 & 1.74 & 0.76 & 0.63 & 0.92 & 0.99 & 0.82 & 1.20 \\
\hline School bus & 0.65 & 0.25 & 1.69 & 1.71 & 0.81 & 3.60 & 0.47 & 0.25 & 0.90 & 0.69 & 0.39 & 1.20 \\
\hline $\begin{array}{l}\text { Delivered by } \\
\text { parents }\end{array}$ & 1.01 & 0.84 & 1.22 & 1.23 & 0.95 & 1.58 & 1.15 & 0.98 & 1.34 & 1.62 & 1.36 & 1.92 \\
\hline \multirow{2}{*}{$\begin{array}{l}\text { Public } \\
\text { transportation }\end{array}$} & \multicolumn{3}{|l|}{ Ref } & \multicolumn{3}{|l|}{ Ref } & \multicolumn{3}{|l|}{ Ref } & \multicolumn{3}{|l|}{ Ref } \\
\hline & Urban & & & Rural & & & Urban & & & Rural & & \\
\hline Walking & 1.21 & 1.03 & 1.42 & 1.25 & 0.95 & 1.64 & 1.11 & 0.98 & 1.26 & 1.33 & 1.08 & 1.65 \\
\hline Cycling & 1.66 & 1.38 & 2.01 & 1.09 & 0.78 & 1.51 & 0.86 & 0.74 & 1.01 & 0.94 & 0.73 & 1.23 \\
\hline School bus & 1.17 & 0.58 & 2.35 & 0.95 & 0.32 & 2.83 & 0.65 & 0.38 & 1.09 & 0.52 & 0.26 & 1.05 \\
\hline $\begin{array}{l}\text { Delivered by } \\
\text { parents }\end{array}$ & 1.17 & 0.98 & 1.38 & 0.77 & 0.56 & 1.04 & 1.36 & 1.19 & 1.55 & 1.30 & 1.03 & 1.65 \\
\hline Public & Ref & & & Ref & & & Ref & & & Ref & & \\
\hline & $\begin{array}{l}\text { Less t } \\
\text { colleg }\end{array}$ & iniversity & & $\begin{array}{l}\text { Colle } \\
\text { highe }\end{array}$ & „nivers & & $\begin{array}{l}\text { Less t } \\
\text { colleg }\end{array}$ & iniversity & & $\begin{array}{l}\text { Colle } \\
\text { highe }\end{array}$ & univers & \\
\hline (Continued) & & & & & & & & & & & & \\
\hline Walking & 1.22 & 1.04 & 1.43 & 1.22 & 0.93 & 1.60 & 1.25 & 1.11 & 1.42 & 0.97 & 0.78 & 1.20 \\
\hline Cycling & 1.50 & 1.24 & 1.82 & 1.53 & 1.12 & 2.09 & 0.90 & 0.77 & 1.05 & 0.82 & 0.63 & 1.07 \\
\hline School bus & 0.97 & 0.45 & 2.09 & 1.29 & 0.52 & 3.24 & 0.53 & 0.32 & 0.90 & 0.70 & 0.33 & 1.48 \\
\hline $\begin{array}{l}\text { Delivered by } \\
\text { parents }\end{array}$ & 0.99 & 0.83 & 1.18 & 1.23 & 0.93 & 1.62 & 1.40 & 1.23 & 1.60 & 1.19 & 0.95 & 1.49 \\
\hline
\end{tabular}

MVPA Moderate to vigorous physical activity, ST Screen time. Bold fonts denote statistical significance. 


\begin{tabular}{|c|c|c|c|c|c|c|c|c|c|c|c|c|}
\hline \multirow{2}{*}{$\begin{array}{l}\text { Public } \\
\text { transportation }\end{array}$} & \multicolumn{6}{|c|}{ MVPA } & \multicolumn{6}{|l|}{ ST } \\
\hline & \multicolumn{3}{|l|}{ Ref } & \multicolumn{3}{|l|}{ Ref } & \multicolumn{3}{|l|}{ Ref } & \multicolumn{3}{|l|}{ Ref } \\
\hline & \multicolumn{3}{|c|}{ Officer worker } & \multicolumn{3}{|c|}{ Manual laborer } & \multicolumn{3}{|c|}{ Officer worker } & \multicolumn{3}{|c|}{ Manual laborer } \\
\hline Walking & 1.19 & 1.01 & 1.41 & 1.30 & 1.01 & 1.69 & 1.17 & 1.02 & 1.33 & 1.18 & 0.98 & 1.42 \\
\hline Cycling & 1.39 & 1.14 & 1.69 & 1.85 & 1.36 & 2.50 & 0.95 & 0.80 & 1.11 & 0.75 & 0.59 & 0.94 \\
\hline School bus & 1.09 & 0.56 & 2.12 & 0.92 & 0.27 & 3.15 & 0.73 & 0.44 & 1.22 & 0.37 & 0.17 & 0.82 \\
\hline $\begin{array}{l}\text { Delivered by } \\
\text { parents }\end{array}$ & 0.99 & 0.83 & 1.18 & 1.27 & 0.96 & 1.69 & 1.33 & 1.16 & 1.53 & 1.38 & 1.12 & 1.70 \\
\hline $\begin{array}{l}\text { Public } \\
\text { transportation }\end{array}$ & \multicolumn{3}{|l|}{ Ref } & \multicolumn{3}{|l|}{ Ref } & \multicolumn{3}{|l|}{ Ref } & \multicolumn{3}{|l|}{ Ref } \\
\hline
\end{tabular}

\section{Discussion}

To the best of our knowledge, this study is one of the first cross-sectional investigations into the relationship of ATS and its types with PA and ST among adolescents of the Chinese samples. This research analyses the correlation of ATS and its types with PA and ST in relation to different individual and parental characteristics, which potentially offers significant practical implications and advances the knowledge in this field.

\section{Key findings}

In this current study, the proportion of the sample engaging in ATS was $47.3 \%$, with participants who walked (34.1\%) constituting a greater percentage than those who cycled (13.2\%). Regarding the question relating to levels of adequate PA and limited ST among the adolescents, only $17.3 \%$ attained the former, whereas the latter was under acceptable levels. Furthermore, we found that participants engaged in ATS had a greater chance of also participating in adequate PA levels, while ATS was not connected with limited ST. Regarding types of ATS, participants engaged in walking or cycling both had a higher chance of undertaking sufficient PA rather than being characterised by limited ST. The relationships of ATS and its types with PA and ST differed according to individual and parental characteristics.

\section{Interpretations of findings}

Low PA levels among adolescents have been demonstrated across the literature $[1,3,30]$. The present research indicated that only approximately $20 \%$ of adolescents participated in the recommended level of PA (60 minutes of moderate to vigorous PA daily [4]), indicating a lower level of PA among adolescents. This result is consistent with the previously published studies of a regional nature [31, 32]. Nevertheless, in contrast with the nationally representative surveys in China [30], the PA level established in the present study is lower. This reduced level is potentially explained by the varying measures adopted, which possibly produced inconsistencies in terms of estimating the amount of PA. Concerning the level of ST among adolescents in this study, our level is in accordance with studies based on Chinese adolescent samples [30-32]. Despite the ST level exceeding 60\%, limiting ST is a necessary mission for the promotion of adolescent health. Likewise, given the health benefits linked to sufficient PA among adolescents [5, 8], increased PA is further imperative. The Social Ecological Model has summarised the correlates of PA and ST [27]. Nevertheless, given the significant variations in China's economy, society and geography, investigating the correlates and determinants of PA and ST among adolescents across the country's various regions requires additional research [31].

The current study indicates that just below half of the participants (47.3\%) engaged in ATS from home to school, which corroborates other studies on Chinese [21,22] and North American samples [34,35]. Regardless, in contrast with studies of European countries [36,37], the adolescents in our study showed reduced ATS performance. Inconsistencies in the ATS levels between our study other research are primarily due to different measures used. Nevertheless, it is evident that the ATS among Chinese adolescents has declined over the past decade [22], suggesting that they are less physically active (through walking or cycling). From this perspective, the ATS level among adolescents in our study is potentially unsatisfactory. This is a somewhat concerning situation that should be explained in relation to local 
contexts and across various regions or cities [38]. Given the health benefits of ATS for adolescents [28], the promotion of ATS among adolescents is to be encouraged.

Our research is one of the few investigations assessing types of ATS among Chinese adolescents. Among the participants engaged in ATS, the majority walked between school and home. This finding is consistent with previous studies [21]. For instance, Yang et al. [22] clarified that participation in walking or cycling between home and school has declined over the years. Presently, there is a dearth of understanding regarding walking or cycling trends among Chinese adolescents at the national or regional level. We suggest that future studies should seek to more effectively explore the patterns of either walking or cycling, thus providing evidence-based information for promoting ATS.

Reflecting the findings of numerous previous studies $[14,19,25,26]$, the current study reaffirms that participants engaged in ATS have a greater chance of participating in sufficient PA. Potential reasons may be that 1) ATS is an aspect of daily PA; thus, participants engaged in ATS are more likely to report higher PA; 2) participants engaged in ATS were similarly or more intensely engaged in other types of PA. Collectively, these two potential reasons may be responsible for the association of ATS with sufficient PA. Nevertheless, the ATS and PA were affected by different variables across various regions.

Accordingly, the mechanism connecting ATS and PA varies depending upon the social and environmental factors. Therefore, it is advocated that further studies should investigate the correlation of ATS and PA to a greater extent, implementing enhanced study designs and multiple data sources. Nevertheless, this study finds that ATS was not associated with ST among adolescents. A systematic review indicated that the relationship of ATS with ST was inconsistent across the literature [12], meaning the correlation between these two variables remains ambiguous. However, informed by the findings of this study, ATS potentially plays a role in promoting PA, as opposed to diminishing ST among adolescents.

We further discovered that the relationship of ATS with PA varies according to sex, age, and parental education. Specifically, the relationship of ATS and PA was found to be significant among boys, suggesting that only boys engaging in ATS are potentially involved in sufficient PA [13]. However, such a significant relationship was not observed among girls. This maybe potentially because parents grant greater freedom to boys to engage in ATS as opposed to girls. Thus, girls may be exposed to fewer opportunities to engage in ATS behaviour, ultimately restricting their ability to engage in sufficient PA levels. Moreover, the sex difference in the relationship of ATS with PA was similar to the age difference.

The present research found that younger adolescents, as opposed to their older peers were engaged more in ATS r and had a greater chance of also participating in sufficient PA. This result is explainable according to the Chinese context. In our study, older adolescents were those in grades 10-12, which is a vital period that covers the college entrance test. During this period, adolescents tend to be delivered to school by their parents for timesaving reasons, allowing a greater amount of time to be spent on studying. Conversely, such a situation would not arise among younger adolescents due to lower academic pressure.

Among participants whose parents had lower educational attainment, those engaged in ATS had a greater prospect of attaining sufficient PA levels. Nevertheless, this significant relationship was not detected among participants whose parents had higher educational attainment levels. A potential reason is that parents with lower levels of educational attainment have lower levels of income compared with their counterparts. Consequently, it is less likely that those parents possess automobiles, which potentially causes their children to travel between home and school through waling or cycling.

This research has established that the relationships between types of ATS with PA and ST vary according to individual and parental characteristics. We primarily identified that participants engaged in walking or cycling had a greater likelihood of participating in sufficient PA compared with their counterparts. Unsurprisingly, when assessing the odds ratio of walking and cycling in relation to sufficient PA, the former is lower than the latter. This finding is consistent with the results of the study of Roth et al. [13], where the association between cycling and overall PA $(\mathrm{OR}=1.93)$ was stronger than that between walking and overall PA $(\mathrm{OR}=1.17)$. Another study also provided a similar finding that there was a difference in the PA level between active walkers and cyclists [39]. However, there is still no well-recognized explanation for this finding and more research is required into the social and environmental determinants of ATS with PA.

A similar relationship was identified among boys, younger adolescents, participants whose parents had reduced education levels, or were office workers. Significantly, only girls engaged in cycling showed sufficient PA in our study, providing an inconsistency with the results for boys. Future studies should attempt to answer this difference for the relationship of types of ATS with PA based on the different subpopulations' characteristics and other factors.

A noticeable distinction is apparent in the relationship of types of ATS with PA according to the geographical location. Specifically, in urban areas, adolescents engaged in cycling were more likely to be involved in sufficient PA, whereas adolescents in rural areas who were engaged

Page $12 / 16$ 
in walking had a greater prospect of undertaking sufficient PA. Unfortunately, no data provided by our study were able to clarify the variation. The differences of built environment between urban and rural settings may provide a plausible explanation to this. A similar finding was established for participants with different parental education levels and occupations. Given the limited evidence across the extant literature, more studies should address these research questions in the future.

When looking at the difference of individual and parental characteristics in the relationship of types of ATS with limited ST, some novel findings should be mentioned. In the current study, participants who walked and were delivered by parents had higher odds of having limited ST compared with their counterparts. To our knowledge, no data provided by previous studies were comparable with our study findings. Some possible reasons for explaining the findings are that 1) participants who walked to school could be regarded as a lower level of socio-economic status, and may not be able to afford as many screen-based devices, ultimately reducing their ST;2) participants delivered by parents would be exposed to much stricter supervision that limits the time spent for screen-based activities. Owing to less research, more studies should confirm our assumptions. In addition to the overall findings, the individual and parental differences in the relationship of types of ATS with ST were found. Such variations should be explained by more factors, whereas the present study failed to provide this indicative information, future studies should answer the variations found by our study, which can provide more specific practical implications.

\section{Implications and future recommendations for research}

Irrespective of the preliminary nature of the evidence regarding the variations in the relationship of ATS and its types with individual and parental characteristics, the present study affirmed the role of ATS in promoting PA among adolescents. This research has expanded on prior research, demonstrating that individual and parental characteristics show different relationships regarding ATS compared to PA. Nevertheless, given that ATS and PA are two complex behaviours that are affected by numerous variables, the relationships of ATS and its types with PA according to various characteristics requires further replication and clarification of the results to provide more robust evidence. Practically, the current study should prove beneficial in encouraging adolescent PA through ATS, with the design of effective policies and actions being necessary.

When designing ATS interventions for enhancing PA among adolescents, individual and parental characteristics must be considered. It is recommended that future research should concentrate on the mechanisms linking ATS with PA within various contexts (for example sex and geographical location). Based on the cross-sectional nature of our research, prospective longitudinal studies are necessary to confirm the relationships we observed, as well as to elucidate whether a potential causal relationship is apparent between ATS and PA. Moreover, it is necessary to undertake further experimental research in order to analyse the extent to which ATS interventions offer further effectiveness for promoting PA among adolescents.

\section{Study strengths and limitations}

This study offered certain advantages. First, the study adopted a relatively large sample size as a means of investigating the relationship of ATS with PA and ST, thus enhancing the generalizability of research findings. Second, the present study is one of the very limited number of investigations analysing the associations of ATS with PA and ST as they relate to different individual and parental characteristics. The research findings are potentially beneficial for designing specific PA and ST interventions. However, there are certain limitations to this study that should be clarified. Due to the cross-sectional nature of the study, the findings of the study should be interpreted with caution. Moreover, our study used a self-reported questionnaire to assess the ATS, PA, and ST, which was subjective to recall bias of measurement. Third, this study did not include/explore more potential confounders, such as time spent during ATS and car ownership, that may affect the relationship of ATS with PA and ST. Fourth, to better explain the relationship of ATS with PA or ST, additional psychological (e.g., attitude towards to ATS), social (e.g., safety and parental awareness of ATS) and physical environmental (e.g., distance to school) factors should be considered for more reliable interpretations. Finally, the ATS and PA, as well as ST, are also affected by other factors, such as income level. We recommend further study of the relationship of ATS with PA and ST by income and other sociodemographic factors, particularly of longitudinal nature. Future studies should address these limitations to provide an improved evidence base.

\section{Conclusions}

Overall, our study indicated that approximately half of adolescents engaged in ATS, with a majority of participants preferring to travel through cycling between home and school. ATS among adolescents was linked with sufficient PA as opposed to ST. Therefore, the relationships of ATS and its types with PA require further clarification in accordance with different contexts, including sex, age differences,

Page $13 / 16$ 
and parental characteristics. Nevertheless, this study retains its specific significance to the implementation of PA promotional activities among adolescents.

\section{Abbreviations}

ATS: Active travel to school; Cl: Confidence interval; HBSC: Health Behavior School-aged Children; IRB: Institutional Review Board; MVPA: Moderate to vigorous physical activity; OR: Odds ratio; PA: Physical activity; ST: screen time; TV: television

\section{Declarations}

\section{Ethics approval and consent to participate}

The survey was approved by the Institutional Review Board (IRB) of the Wuhan University of Technology. All participants provided written informed consent before the start of the investigation.

\section{Consent for publication}

Not applicable.

\section{Availability of data and materials}

The datasets analysed in this study are available from the corresponding author on reasonable request.

\section{Competing interests}

The authors declare that they have no competing interests.

\section{Funding}

The study did not receive any external funding.

\section{Authors' contributions}

$\mathrm{CH}, \mathrm{YL}, \mathrm{YL}$ and STC conceptualised and designed this study. $\mathrm{CH}, \mathrm{CL}$ and $\mathrm{YL}$ analysed, interpreted data, and drafted the manuscript. ARM and STC provided important intellectual roles in revision. All authors read and approved the final manuscript.

\section{Acknowledgements}

The authors expressed thanks to the participants in this study.

\section{Author details}

${ }^{1}$ School of Physical Education, Hubei University of Technology, Wuhan 430068, China. ${ }^{2}$ Institute for Health and Sport, Victoria University, Melbourne 3001, Victoria, Australia. ${ }^{3}$ Library of Beijing Sport University, Beijing 100084, China. ${ }^{4}$ Shanghai Research Institute of Sports Science, Shanghai 200030, China. ${ }^{5}$ Department of Physical Education, Wuhan University of Technology, Wuhan 430070, China

\section{References}

1. Hallal PC, Andersen LB, Bull FC, Guthold R, Haskell W, Ekelund U. Lancet Phys Activity Series W. Global physical activity levels: surveillance progress, pitfalls, and prospects. Lancet. 2012;380:247-57. 
2. Tremblay MS, Barnes JD, Gonzalez SA, Katzmarzyk PT, Onywera VO, Reilly JJ, Tomkinson GR. Global matrix 2.0: report card grades on the physical activity of children and youth comparing 38 countries. J Phys Act Health. 2016;13:343-66.

3. Guthold R, Stevens GA, Riley LM, Bull FC. Global trends in insufficient physical activity among adolescents: a pooled analysis of 298 population-based surveys with 1.6 million participants. Lancet Child Adolesc Health. 2020;4(1):23-35.

4. Tremblay MS, Carson V, Chaput J-P, Gorber SC, Thy D, Duggan M, et al. Canadian 24-hour movement guidelines for children and youth: an integration of physical activity, sedentary behaviour, and sleep. Appl Physiol Nutr Metab. 2016;41:311-27.

5. Janssen I, LeBlanc AG. Systematic review of the health benefits of physical activity and fitness in school-aged children and youth. Int $J$ Behav Nutr Phys Act. 2010;7:40-55.

6. Tremblay MS, LeBlanc AG, Kho ME, Saunders TJ, Larouche R, Colley RC, Goldfield G, Gorber SC. Systematic review of sedentary behaviour and health indicators in school-aged children and youth. Int J Behav Nutr Phys Act. 2011;8:98-119.

7. Carson V, Hunter S, Kuzik N, Gray CE, Poitras VJ, Chaput J-P, Saunders TJ, Katzmarzyk PT, Okely AD, Gorber SC, et al. Systematic review of sedentary behaviour and health indicators in school-aged children and youth: an update. Appl Physiol Nutr Metab. 2016;41:240-65.

8. Poitras VJ, Gray CE, Borghese MM, Carson V, Chaput J-P, Janssen I, Katzmarzyk PT, Pate RR, Gorber SC, Kho ME, et al. Systematic review of the relationships between objectively measured physical activity and health indicators in school-aged children and youth. Appl Physiol Nutr Metab. 2016;41:197-239.

9. Chaput JP, Leduc G, Boyer C, Bélanger P, LeBlanc AG, Borghese MM, Tremblay MS. Objectively measured physical activity, sedentary time and sleep duration: independent and combined associations with adiposity in Canadian children. Nutr Diabetes. 2014;4:e117.

10. Biddle SJH, Connell S, Braithwaite RE. Sedentary behaviour interventions in young people: a meta-analysis. Br J Sports Med. 2011;45:937-42.

11. Heath GW, Parra DC, Sarmiento OL, Andersen LB, Owen N, Goenka S, Montes F, Brownson RC. Evidence-based intervention in physical activity: lessons from around the world. Lancet. 2012;380:272-81.

12. Schoeppe S, Duncan MJ, Badland H, Oliver M, Curtis C. Associations of children's independent mobility and active travel with physical activity, sedentary behaviour and weight status: a systematic review. J Sci Med Sport. 2013;16:312-19.

13. Roth MA, Millett CJ, Mindell JS. The contribution of active travel (walking and cycling) in children to overall physical activity levels: a national cross-sectional study. Prev Med. 2012;54:134-39.

14. Barranco-Ruiz Y, Guevara-Paz AX, Ramírez-Vélez R, Chillón P, Villa-González E. Mode of commuting to school and its association with physical activity and sedentary habits in young ecuadorian students. Int J Environ Res Public Health. 2018;15:2704-15.

15. Sahlqvist S, Song Y, Ogilvie D. Is active travel associated with greater physical activity? The contribution of commuting and noncommuting active travel to total physical activity in adults. Prev Med. 2012;55:206-11.

16. Nilsson A, Andersen LB, Ommundsen Y, Froberg K, Sardinha LB, Piehl-Aulin K, Ekelund U. Correlates of objectively assessed physical activity and sedentary time in children: a cross-sectional study (The European Youth Heart Study). BMC Public Health. 2009;9:322-8.

17. Heelan KA, Donnelly JE, Jacobsen DJ, Mayo MS, Washburn R, Greene L. Active commuting to and from school and BMI in elementary school children-preliminary data. Child Care Health Dev. 2005;31:341-9.

18. King AC, Parkinson KN, Adamson AJ, Murray L, Besson H, Reilly JJ, Basterfield L, Team GMSC. Correlates of objectively measured physical activity and sedentary behaviour in English children. Eur J Public Health. 2011;21:424-31.

19. Owen CG, Nightingale CM, Rudnicka AR, Van Sluijs EM, Ekelund U, Cook DG, Whincup PH. Travel to school and physical activity levels in 9-10 year-old UK children of different ethnic origin; child heart and health study in England (CHASE). PloS One. 2012;7:e30932.

20. Landsberg B, Plachta-Danielzik S, Much D, Johannsen M, Lange D, Müller MJ. Associations between active commuting to school, fat mass and lifestyle factors in adolescents: the Kiel Obesity Prevention Study (KOPS). Eur J Clin Nutr. 2008;62:739-47.

21. Sun Y, Liu Y, Tao FB. Associations between active commuting to school, body fat, and mental well-being: population-based, crosssectional study in China. J Adolesc Health. 2015;57:679-85.

22. Yang Y, Hong X, Gurney JG, Wang YF. Active travel to and from school among school-age children during 1997-2011 and associated factors in China. J Phys Act Health. 2017;14:684-91.

23. Zacharias J, Zhen B, Han X, Huang Y. Local environment and social factors in primary school children's afterschool commute in China. Prev Med Rep. 2017;7:206-10.

24. Meng S, Zacharias J, Han X. Longer afterschool active commutes and the travel environment of middle schools in Shenzhen, China. Prev Med Rep. 2018;12:170-5.

25. Gao Y, Wang JJ, Lau PWC, Ransdell L. Pedometer-determined physical activity patterns in a segmented school day among Hong Kong primary school children. J Exerc Sci Fit. 2015;13:42-8.

Page 15/16 
26. Huang WV, Wong SH, He G. Is a change to active travel to school an important source of physical activity for Chinese children? Pediatr Exerc Sci. 2017;29:161-8.

27. Bauman AE, Reis RS, Sallis JF, Wells JC, Loos RJF, Martin BW. Correlates of physical activity: why are some people physically active and others not? Lancet. 2012;380:258-71.

28. Lubans DR, Boreham CA, Kelly P, Foster CE. The relationship between active travel to school and health-related fitness in children and adolescents: a systematic review. Int J Behav Nutr Phys Act. 2011;8:1-12.

29. Liu Y, Wang M, Tynjälä J, Lv Y, Villberg J, Zhang Z, Kannas L. Test-retest reliability of selected items of Health Behaviour in School-aged Children (HBSC) survey questionnaire in Beijing, China. BMC Med Res Methodol. 2010;10:73-81.

30. Zhu Z, Tang Y, Zhuang J, Liu Y, Wu X, Cai Y, Wang L, Cao Z-B, Chen P. Physical activity, screen viewing time, and overweight/obesity among Chinese children and adolescents: an update from the 2017 physical activity and fitness in China-the youth study. BMC Public Health. 2019;19:197-204.

31. Chen S-T, Liu Y, Hong J-T, Tang Y, Cao Z-B, Zhuang J, Zhu Z, Chen P-J. Co-existence of physical activity and sedentary behavior among children and adolescents in Shanghai, China: do gender and age matter? BMC Public Health. 2018;18:1287-95.

32. Liu Y, Tang Y, Cao ZB, Chen PJ, Zhang JL, Zhu Z, Zhuang J. Results from Shanghai's (China) 2016 report card on physical activity for children and adolescents. J Phys Act Health. 2016;13:124-8.

33. Chillón P, Evenson KR, Vaughn A, Ward DS. A systematic review of interventions for promoting active transportation to school. Int J Behav Nutr Phys Act. 2011;8:10-26.

34. Bookwala A, Elton-Marshall T, Leatherdale ST. Factors associated with active commuting among a nationally representative sample of Canadian youth. Can J Public Health-Rev Can Sante Publ. 2014;105:e348-53.

35. Aubert S, Barnes JD, Abdeta C, Nader PA, Adeniyi AF, Aguilar-Farias N, Andrade Tenesaca DS, Bhawra J, Brazo-Sayavera J, Cardon G, et al. Global matrix 3.0 physical activity report card grades for children and youth: results and analysis from 49 countries. J Phy Act Health. 2018;15:251-73.

36. Pizarro AN, Schipperijn J, Andersen HB, Ribeiro JC, Mota J, Santos MP. Active commuting to school in Portuguese adolescents: using PALMS to detect trips. J Transp Health. 2016;3:297-304.

37. Dessing D, de Vries SI, Graham JMA, Pierik FH. Active transport between home and school assessed with GPS: a cross-sectional study among Dutch elementary school children. BMC Public Health. 2014;14:1-8.

38. Panter JR, Jones AP, van Sluijs EM. Environmental determinants of active travel in youth: a review and framework for future research. Int J Behav Nutr Phys Act. 2008;5:34-47.

39. Cooper AR, Andersen LB, Wedderkopp N, Page AS, Froberg K. Physical activity levels of children who walk, cycle, or are driven to school. Am J of Prev Med. 2005;29:179-84.

\section{Supplementary Files}

This is a list of supplementary files associated with this preprint. Click to download.

- AddtionalfileSTROBEchecklist.docx 